\title{
Sehschärfenuntersuchungen mit Hilfe des Visometers von Zeiss.
}

(Zugleich ein Beitrag zur Frage der Lesbarkeit von Druckschriften.)

Von

Dr. Robert Kirsch,

Augenarzt in Sagan, früher Assistent der Universitüta-Augenklinik, Berlin (Prof. K I ü ekma a n).

Mit 6 Textabbildungen.

Bei einem längeren Aufenthalt in Jena*) im Winter 1913/14 hatte ich Gelegenheit, einen neuen Sehschärfe-Meßapparat der Firma Zeiss kennenzulernen und die ersten Versuche damit anzustellen. Die Eigentümlichkeit des Apparates besteht darin, daß er dem Auge als Sэhobjekt ein in genau einstellbarer ziemlich naher Entfernung gelegenes virtuelles Bild darbietet, welches, ohne seinen Abstand vom Auge zu ändern, stetig und jederzeit meßbar vergrößert oder verkleinert werden kann. Das Instrument ist aus dem Wunsche entstanden, mittels eines handlichen und nicht zu teueren Gerätes die natürliche Sehschärfe genauer zu messen, als es mit den sonst dafür empfohlenen Verfahren möglich ist. Durch stetige Größenänderung der Sehprobe und genaue Ablesbarkeit der Entfernung zwischen ihr und dem vorderen Augenpol kann der Hauptpunktswinkel so sicher festgelegt werden, als es die Natur des Versuchs am Lebenden überhaupt gestattet.

Bevor ich auf eine nähere Beschreibung des Instrumentes eingehe, halte ich es für erforderlich, den Begriff der Sehschärfe noch einmal kurz auseinanderzusetzen; denn auf diesem Gebiete besteht vielfach noch eine bedauerliche Unklarheit, hauptsächlich deshalb, weil die einzelnen Begriffe im Laufe der Zeit von den Autoren in ganz verschiedenem Sinne gebraucht worden sind.

Um von der Leistung des unkorrigierten Auges auszugehen, so sollte diese, welche ohne Rücksicht auf Abweichungen der Refraktion oder Akkommodation sowie auf Unregelmäßigkeiten der Form und Durchlässigkeit der optischen Medien festgestellt wird, am besten überhaupt nicht als "Sehschärfe", sondern stets nur als "Sehleistung“" bezeichnet werden. Diese sehr brauchbare Benennung ist von Trie pel') 1894 (im Anschluß an die Deutsche Marine-Ordnung vom 19. November 1889) in Vorschlag gebracht worden, hat sich aber immer noch nicht restlos eingebürgert. Will man hierfür doch auf den Ausdruck

*) Der Verfasser erhielt dazu eine Unterstützung aus der Gräfin-Bose-Stiftung. v. Graefes Archiv für Ophthalmologie, Bd. 103. 
"Sehschärfe" nicht verzichten, so muß dazu unbedingt ein ganz unzweideutiges Beiwort verwendet werden, etwa, ,unkorrigierte Sehschärfe". Leber ${ }^{2}$ ) gebraucht in diesem Sinne ,wahre oder wirkliche* Sehschärfe (im Gegensatz zu ,korrigierte" Sehschärfe). Der Ausdruck hat sich nicht eingeführt und ist für sich allein auch nicht klar genug, um ohne weiteres verstanden zu werden. Fuchs') (Seite 804) benutzt den Ausdruck, ,relative Sehschärfe"s. Dies muß unweigerlich zu Mißverständnissen führen, demn sowohl im Sinne von Donders wie in dem jetzt wohl allgemein angenommenen von Gull. strand ist ,relative ${ }^{\natural 6}$ Sehschärfe etwas ganz anderes, wie dies weiterhin auseinandergesetzt wird. Ferner behauptet Löh ner ${ }^{4}$ ), daß von Gullstrand der Ausdruck, ,natürliche Sehschärfe" gleichbedeutend mit unserem Begriff ,Sehleistung" verwandt werde. Dies ist ein Irrturn. Was Gullstrand unter ,natürlicher Sehschärfe versteht, wird gleichfalls weiter unten erörtert.

Das Gebiet der Sehleistung, also der nicht durch optische Hilfsmittel erhöhten Leistungsfähigkeit des Auges, ist verhältnismäßig selten be arbeitet worden. Am wichtigsten sind die Veröffentlichungen von Salzmann $n^{5}$ ) über ,Das Sehen in Zerstreuungskreisen", sowie die angeführte Arbeit von Trie pel ${ }^{1}$ ).

Im Gegensatz zur Sehleistung spricht man von ,Sehschärfe" dann, wenn bei der Prüfung die von den optischen Medien entworfenen Bilder der AuBenwelt in die Netzhautebene zu liegen kommen. Sehr treffend definiert Wolffberg ${ }^{6}$ ) dementsprechend die Sehschärfe als die Sehleistung des dioptrisoh normalen oder dioptrisch ausgeglichenen Auges. Tatsächlich unterscheidet sich zwar das Sehen in Zerstreuungskreisen von dem bei genauer Einstellung des Auges nur dem Grade nach, nicht grundsätzlich; denn auch bei letzterem bandelt es sich strenggenommen nicht um punktförmige, sondern um flächenförmige Abbildung punktförmiger Objekte. [Hess7) S.137]. Trotzdem muß der Gegensatz scharf betont werden. Daß für ein emmetropisches Auge im Ruhezustande Sehleistung und Sehschärfe zusammenfallen, ist ohne weiteres klar.

Ehe ich auf die Unterscheidung zwischen absoluter, natürlicher und relativer Sehschärfe eingehe, möchte ich noch kurz zusammenfassen, daß das Maß der Sehschärfe gegeben wird durch die kleinsten Gesichts- oder Distinktionswinkel, unter denen Gegenstände der AuBenwelt eben noch erkannt werden, und zwar ist die Sehschärfe diesen umgekehrt proportional. Dabei handelt es sich entweder um die Wahrnehmung eines feinsten Lageunterschiedes (optischer Raumsinn) oder aber eines Zwischenraumes zwischen zwei Objektpunkten (optisches Auflösungsvermögen). Dieser Unterschied, der zuerst von Hering ${ }^{8}$ ) klar erkannt wurde, braucht uns hier nicht weiter zu beschäftigen. 
Die klassische Norm für die Sehschärfe ist seit Snellen die Bogenminute, indem angenommen wurde, da $B$ unter günstigen Bedingungen zwei Punkte oder Linien, die um 1' voneinander entfernt liegen, vom normalen Auge noch als getrennt erkannt werden müssen (minimum separabile). Der Wert dieser Snellenschen Einheit wird zwar dadurch. daß viele Augen danach eine Sehschärfe $>1$ haben, nicht wesentlich gemindert, doch sollte man sich gewöhnen, stets nur von Sehschärfe $=1$, nicht von ,,normaler" Sehschärfe zu sprechen.

Innerhalb des Sehschärfe-Begriffes unterscheidet man absolute, natürliche und relative Sehschärfe.

Absolute Sehschärfe.

Der Begriff der ,absoluten" Sehschärfe steht seit Donders ${ }^{9}$ ) fest. Donders versteht darunter die Sehschärfe des emmetropischen Auges bei Akkommodationsruhe, ohne Gläser, oder aber die Sehschärfe eines ametropischen Auges, für die Ferne, mit Gläserkorrektion, also kurz:

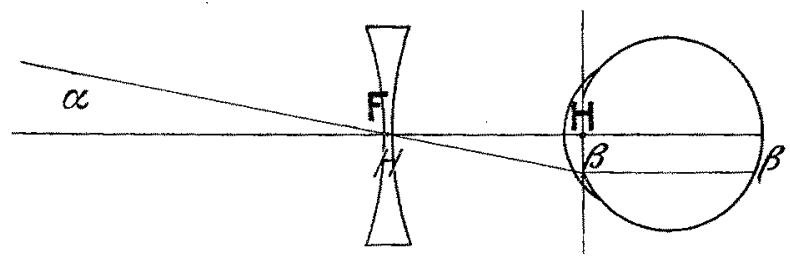

Abb. 1 .

die bei genauer Einstellung und Akkommodationsruhe an weit entfernten Objekten ermittelte Sehschärfe. Leber ${ }^{2}$ ) schlug statt dessen ,korrigierte" Sehschärfe vor, ein Ausdruck, gegen den nichts einzuwenden ist. Die Feststellung der absoluten Sehschärfe ist nach Gullstrand ${ }^{10}$ ), dem wir die endgültige und exakte Fixierung des Begriffs verdanken, geeignet, ein Urteil über die Funktionstüchtigkeit der Netzhaut zu ermöglichen, weil sie verschiedene Augen miteinander zu vergleichen gestattet. Die Messung muß hierzu so ausgeführt werden, daß in Augen von verschiedener Achsenlänge von demselben Objekt gleich große, dem emmetropischen Auge entsprechende Netzhautbilder entstehen. Diese Forderung wird erfüllt, wenn der Gesichtswinkel im vorderen Augenbrennpunkt $\mathbf{F}$ gemessen und wenn bei Ametropie das korrigierende Glas so vor das Auge gebracht wird, daß dessen (zweiter) Hauptpunkt mit dem vorderen Brennpunkt des Auges zusammenfällt. Denn nur so kann der den vorderen Augenbrennpunkt schneidende Strahl mit einer durch das Brillenglas nicht veränderten Neigung in die Augenhauptebene einfallen.

Die Abbildung 1 erläutert den ungebrochenen Durchgang des Strahls durch das Zentrum des (dünn angenommenen) Brillenglases und die 
Entstehung des telezentrischen (achsenparallelen) Strahlengangs im Augeninneren, der dadurch gegeben wird, daß der Strahl im Brillenzentrum $H$ zugleich auch den vorderen Augenbrennpunkt $\mathrm{F}$ schneidet. Infolge dieses achsenparallelen Strahlenganges wird die Bildgröße von der Achsenlänge des Auges unabhängig.

Die eben dargestellten Verhältnisse werden gewöhnlich mathematisch abgeleitet, und zwar folgendermaßen:

Gleiche Bildgröße im unbewaffneten und im bewaffneten Auge besteht dann, wenn beide gleiche Gesamtbrechkraft haben, also, wenn die Brechkraft des Auges $\left(D_{11}\right)$ gleich ist der Brechkraft des aus Auge und Brille zusammengesetzten Systems $\left(D_{12}=D_{1}+D_{11}-\delta D_{1} D_{11}\right)$.

Also wenn

$$
\begin{aligned}
& D_{11}=D_{12}=D_{1}+D_{11}-\delta D_{1} D_{11} \\
& D_{11}=D_{11}+D_{1}\left(1-\delta D_{11}\right) .
\end{aligned}
$$

Die Gleichung wird erfüllt, wenn entweder $D_{1}(=$ Brechkraft des Glases, also im Fall des unbewaffneten Auges) oder wenu $\left(1-\delta D_{11}\right)=0$ werden. Aus letzterem folgt

$$
\delta(=\text { Abstand des Brillenglases })=\frac{1}{D_{11}}=f .
$$

Demmach muß das korrigierende Glas um f vor dem Augenhauptpunkt, also in $F$ stehen.

\section{Natürliche Sehschärfe.}

Der Begriff der ,natürlichen" Sehschärfe stammt von Gullstrand ${ }^{10}$ ). Sie soll, ,ein Maß der Funktionstüchtigkeit des individuellen Auges" ergeben, ,welches vom Akkommodationszustand unabhängig sein, mithin durch den kleinsten Hauptpunktswinkel gemessen werden nuBs: Denn während der vordere Brennpunkt $F$ bei der Akkommodation, entsprechend dem großen Zuwachs an Brechkraft, sehr erhebliche Verschiebungen erleidet, verschiebt sich der hintere Augenhauptpunkt $H^{\prime}$ nach den Werten für das Gullstrandsche exakte schematische Auge bei einer Akkommodationsbreite von 10,6 dptr nur um $0,484 \mathrm{~mm}$, einen Betrag, der gut vernachlässigt werden kann. Die Feststellung der natürlichen Sehschärfe erfolgt also am unbewaffneten, gleichgültig, ob akkommodierenden oder nicht akkommodierenden Auge, wobei die Abbildung in der Netzhautebene erfolgen muB, also bei Einstellung auf ein Objekt, das zwischen Ferm- und Nahpunkt des Auges gelegen ist. Für ein ruhendes emmetropisches Auge ergeben demnach absolute und natürliche Sehschärfe gleiche Werte. Für ametrope Augen dagegen besteht keine Ủbereinstimmung, denn ein myopisches Auge ergibt bei der Prüfung in der Nähe dank seiner großen Achsenlänge größere Netzhautbilder und entsprechend größere Sehschärfe als bei Prüfung in der Ferne mittels Gläserkorrektion im vorderen Brenn- 
punkt, wobei wie angeführt, die Netzhautbilder denen eines emmetropischen Auges von normaler Achsenlänge entsprechen. Und ein hyperopisches Auge liefert unkorrigiert, für die Ferne akkommodierend, bei seiner kleinen Achsenlänge kleinere Netzhautbilder und damit kleinere Sehschärfenwerte, als wenn es korrigiert wird, wobei auch hier die Bilder dem des emmetropen Auges gleich groß werden. Die natürliche Sehschärfe steigt und fällt also (bei gleicher Funktionstüchtigkeit der Netzhaut) mit der Achsenlänge.

Die Ableitung der Formeln für das Verhältnis zwischen absoluter und natürlicher Sehschärfe, wie sie u. a. bei Hess') (S. 202-205) zu finden ist, soll hier nicht wiedergegeben werden. Die Formeln lauten:

$$
S_{n}=S \cdot\left(1-\frac{c}{100} D\right)
$$

und

$$
S=S_{n} \cdot\left(1+\frac{e}{100} A\right)
$$

worin $c$ den in Zentimetern gemessenen Abstand des Brillenglases vom ersten Augenhauptpunkt (abgerundet 1,5 cm; Hess hat entgegengesetzte Vorzeichen, da er $c$ negativ annimmt), $D$ die Brechkraft des Korrektionsglases und $A$ den Ametropiegrad des Auges bezeichnet. Die Formeln besagen in Worten: „Man erhält die natürliche Sehschärfe aus der absoluten, indem man von letzterer für jede Dioptrie des benutzten Korrektionsglases 1,5\% bei Hypermetropie abzieht, bei Myopie zu ihr zuzählt. Man erhält die absolute Sehschärfe aus der natürlichen, indem man für jede Dioptrie Ametropie $1,5 \%$ bei Hypermetropie zu ihr zuzählt, bei Myopie von ihr abzieht."

\section{Relative Sehschärfe.}

Der Ausdruck ,relative" Sehschärfe bildet in jedem Falle einen Gegensatz zur absoluten Sehschärfe. (Daß er von Fuchs im Sinne von „,Sehleistung" gebraucht wurde, hatten wir schon erwähnt.) Der.Gegensatz zur absoluten Sehschärfe wurde aber von den früheren Autoren im Anschluß an Donders anders gefaßt als später von Gullstrand. Donders ${ }^{9}$ ) nämlich versteht darunter die in der Nähe bestimmte Sehschärfe, im Falle von Emmetropie oder Hyperopie bei Einstellung. mit Hilfeder Akkommodation oder durch Gläser, bei Myopen eventuell im Fernpunkt. Seine relative Sehschärfe ist also ungefähr (aber nicht genau) dasselbe, was wir jetzt natürliche Sehschärfe nennen. Gullstrand dagegen mißt die relative Sehschärfe ebenso wie die absolute beim Blick in die Ferne unter Gläserkorrektion. Aber während bei der absoluten Sehschärfe, wie wir sahen, die Bedingung erfüllt war, daß der zweite Hauptpunkt des Brillenglases mit dem vorderen Augen- 
brennpunkt zusammenfällt, ergibt eine Vernachlässigung dieser Bedingung die relative Sehschärfe. Die Messung des Distinktionswinkels erfolgt dann in dem vorderen Hauptpunkt des vorgeschalteten Systems.

Die Richtung, nach der die relativen Sehschärfe-Werte von denen der absoluten abweichen, ergibt sich aus folgender Überlegung: Die Netzhautbildgröße eines ametropischen Auges bleibt bei Korrektion nur dann unverändert, wenn diese ohne Verschiebung der Hauptpunkte möglich ist (wie es angenähert bei der natürlichen Korrektion, der Akkommodation, geschieht). Die gewöhnliche Art der Korrektion dagegen verändert die Netzhautbildgrößen: Wir sahen vorhin, daß die verhältnismäßig großen Netzhautbilder eines myopischen Auges durch Korrektion im vorderen Augenbrennpunkt auf die Guöße emmetropischer Augen verkleinert, die verhältnismäßig kleinen Bilder hyperopischer Augen durch ebensolche Korrektion bis zur Größe emmetropischer Augen vergrößert wurden. Korrektion in größerem Abstande als f wird also bei Myopie weitere Verkleinerung, bei Hyperopie weitere Vergrößerung, Korrektion in kleinerem Abstande als $f$ bei Myopie relative (gegenüber den Netzhautbildern exmetropischer Augen als Maß der absoluten Sehschärfe) Vergrößerung, bei Hyperopie relative Verkleinerung ergeben. Also die relative Sehschärfe wird, wenn der Abstand des Brillenglases $>f$, bei Myopie kleiner, bei Hyperopie größer als die absolute Sehschärfe gefunden, ist der Abstand dagegen < $f$, bei Myopie größer, bei Hyperopie kleiner. Ist der Abstand $=f$, so wird die relative Sehschärfe gleich der absoluten, erstere ist also gewissermaßen der allgemeinere, letztere der spezielle Fall der korrigierten Sehschärfe. Dementsprechend gelten die oben gegebenen Formeln auch für das Verhältnis zwischen natürlicher und relativer Sehschärfe und lauten nach von $\operatorname{Rohr}^{11}$ ) (S. 21)

und

$$
\begin{aligned}
& S_{n}=S_{r} \cdot\left(1-\delta D_{1}\right) \\
& S_{r}=S_{n} \cdot(1+\delta A) .
\end{aligned}
$$

Die erste Formel enthält die von Gullstrand gegebene Regel : ,Wenn $\delta$ (der Abstand des Brillenglases vom vorderen Augenhauptpunkt) in $\mathrm{cm}$ gemessen wird, so erhält man die natürliche Sehschärfe allgemein aus der bei großem Objektabstande ermittelten relativen Sehschärfe durch Abziehen von $\delta \%$ für jede Dioptrie des angewendeten Glases" (bei Myopie ist Abziehen gleichbedentend mit numerischem Zuzählen). Die zweite Formel hat nur insofern praktische Bedeutung, als sie auch zur Ermittelung der absoluten Sehschärfe aus der natürlichen dient; die relative Sehschärfe wird im allgemeinen nicht gesucht werden. Das Verhältnis zwischen relativer und absoluter. Sehschärfe wird nach Qullstrand ausgedrückt durch die Formel

$$
S_{r}=S \cdot\left(1+\delta_{j} L\right)
$$


worin $\delta_{f}=H^{\prime} \mathrm{F}$ den Abstand zwischen hinterem Brillenhauptpunkt und vorderem Augenbrennpunkt, $L=\frac{1}{\mathrm{~F} O^{\prime}}$ den reziproken Wert des Abstandes zwischen vorderem Augenbrennpunkt und Fernpunkt, also den für den vorderen Brennpunkt geltenden Korrektionswert der Ametropie bedeutet (s. Abb. 2).

Nach diesen einleitenden Bemerkungen komme ich zur Beschreibung des Zeissschen Sehschärfemessers (V i s o meter).

Die bereits oben ausgesprochene Forderung einer stetigen Größenänderung des Sehobjektes bei gleichbleibender Entfernung ließ sich, wenn man wie bei Sehschärfebestimmungen üblich $5 \mathrm{~m}=\infty$ annimmt, zwar durch einfaches Annähern oder Entfernen auf einer Art Schlitten, dem Sehprobenschlitten, erfüllen, doch war diese Vorrichtung zu sperrig und hätte die natürliche Sehschärfe nur bei Emmetropen (bei denen sie mit der absoluten übereinstimmt) zu méssen gestattet. Außerdem ließ sich auf diese Weise nur eine ziemlich geringe Größenänderung erreichen, während èin merklich größerer Spielraum in der Maßstabsänderung erwünscht war,



Abb. 2. so etwa, daß man günstigenfalls imstande wäre, die Sehprobe von $x$ bis $0,1 \quad x$ zu verkleinern.

Der Weg, auf dem man für kurzsichtige Augen, deren Nahpunkt in endlichem Abstand vor dem Auge liegt, diese Ziele verwirklichte, war der folgende. Beachtet man, daB, ganz allgemein gesprochen, eine Linsenfolge von zerstreuender Wirkung einen greifbaren Gegenstand stets verkleinert abbildet, so kann man den Maßstab der Verkleinerung $m$ und die Brechkraft $D_{1}$ als bekannt voraussetzen und gewinnt aus der Formel für Objekt- und Bild-Größe $(\alpha \cdot A=\beta \cdot B)$

$$
\frac{\beta}{\alpha}=m=\frac{A_{1}}{B_{1}}=\frac{A_{1}}{A_{1}+D_{1}}(\text { nach der Formel } B=A+D) ;
$$

daraus folgt

$$
\begin{gathered}
m A_{1}+m D_{1}=A_{1}, \\
m D_{1}=(1-m) \cdot A_{1}, \\
A_{1}=\frac{m}{1-m} \cdot D_{1},
\end{gathered}
$$

d. h. man kann $A_{1}$ aus $m$ und $D_{1}$ berechnen. Setzt man nun, um den Spielraum $x$ bis $0,1 *$ zu gewinnen, einmal $m \doteq x$ und das andere 
Mal $m=\frac{x}{10}$, so erhält man beim ersten Male

$$
A_{1,1}=\frac{x D_{1}}{1-x}
$$

und beim zweiten Male

$$
A_{1,0.1}=\frac{{ }^{\varkappa} D_{1}}{1-\frac{\varkappa}{10}}=\frac{\varkappa D_{1}}{10-x} .
$$

Durch eine zweckmäBige Wahl von $x_{\text {und }} D_{1}$ für $A_{1,1}$ und $A_{1,0.1}$ lassen sich leicht Grenzen finden, die der Vorrichtung handliche Werte geben.

Bei den gewöhnlichen optischen Hilfsmitteln steht die Linse meistens fest, während Ding und Bild ihr gegenüber beweglich sind.

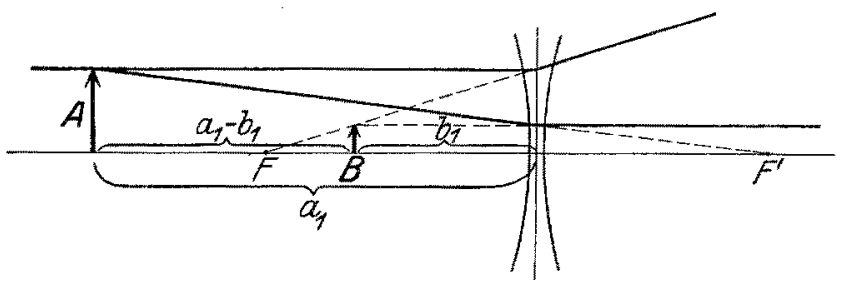

Abo. 8.

Das ist hier nicht erwünscht, vielmehr soll ja nach der oben angegebenen Forderung das Bild, d.h. die stetig verkleinerungsähige Sehprobe gegen das zu prüfende Auge eine feste Lage haben. Mithin sind Ding und Linse so zu verschiehen, daB sich stets die vorgeschriebenen $\mathrm{Ab}$ stände $a_{1}-b_{1}$ und $b_{1}$ von der unveränderlichen Ebene des Bildes ergeben.

Sind für eine Reihe von Verkleinerungen zwischen $A_{1,1}$ und $A_{1,0.1}$ diese Werte berechnet, so lassen sich für eine zwangsläufige Bewegung von Ding und Linse Vorrichtungen schaffen, die mit ausreichender Genauigkeit arbeiten.

Man hat dann weiter nichts zu tun, als den Abstand $a$ zwischen der Bildebene und dem vorderen Augenhauptpunkt des Prüflings festzustellen, was nach seinem Nahepunktsabstande geschehen mag, da er nach der Voraussetzung imstande ist, auf einen wirklichen Gegenstand in endlicher Entfernung einzustellen. Für übersichtige Augen kann man eine sammelnde Hilfslinse verwenden, die man aus dem Brillenkasten so wählt, daß sie den Nahepunkt vor das Auge in eine endliche Entfernung verlegt; doch würde es natürlich an sich auch möglich sein, für übersichtige Augen mit positivem Nahepunkts- 
abstande ein entsprechendes Prüfungsgerät mit einem Glase von sammelnder Wirkung vorzusehen.

Die beigefügte Abbildung 4 zeigt die äußere Form des Instruments. $L$ ist das Linsensystem, welches ebenso wie der Objektträger $O$ auf einer dreikantigen Schiene beweglich ist. Die Verschiebung des Linsenträgers erfolgt durch eine Zugfeder, welche einen unten an ihm angebrachten Stift fest gegen das graduierte Schneckenrad $S$ drückt.

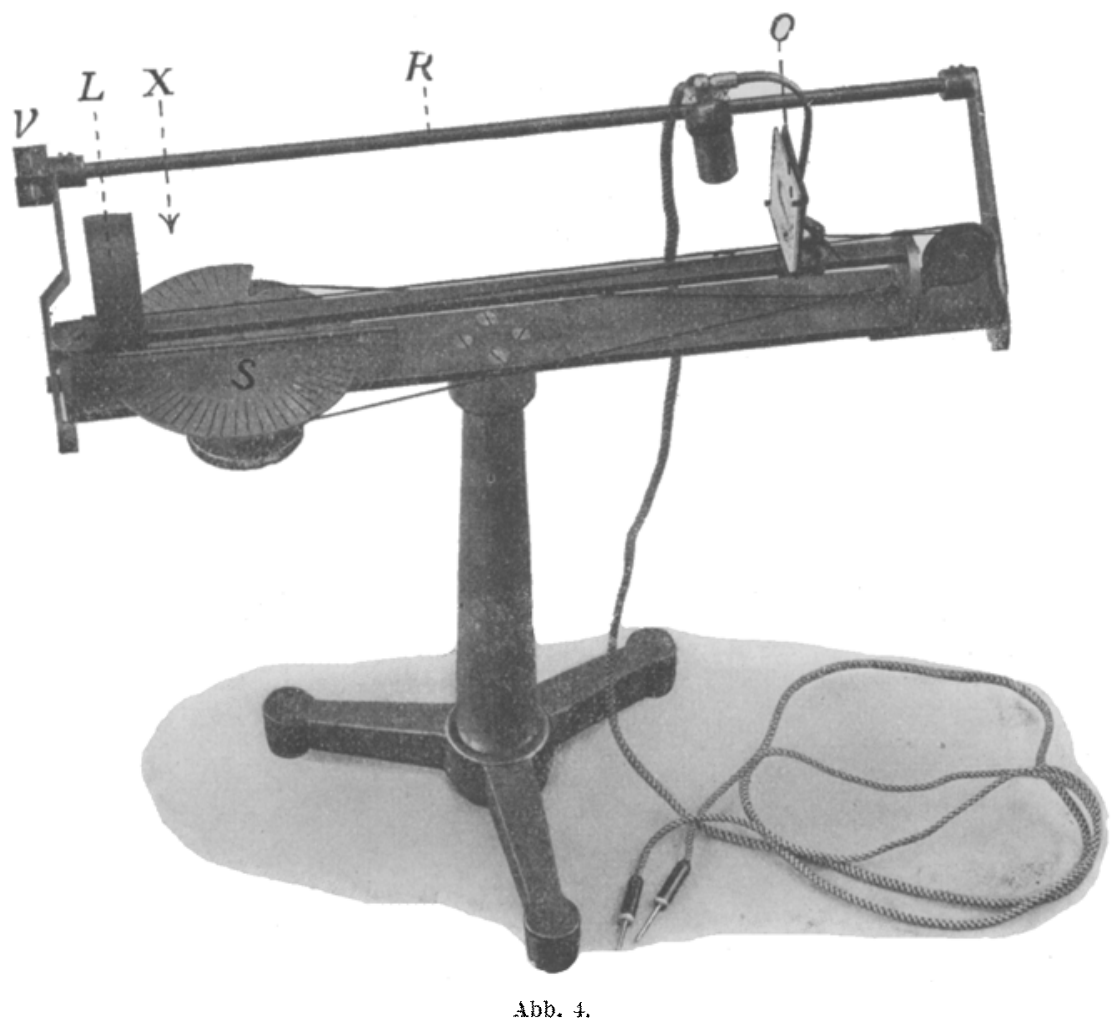

Die mit der Hand bewirkten Drehungen dieses Rades werden dureh eine an seiner Unterseite befestigte Schnurscheibe und die in der $\mathrm{Ab}$ bildung deutlich erkennbaren Schnüre und Führungsrollen auf den Objektträger übertragen, so daß also jeder Verschiebung des Linsensystems eine bestimmte, in ihrer Größe durch die Rechnung festgelegte Bewegung des Objektträgers entspricht.

Da der weitest mögliche Abstand zwischen Objekt und Linsensystem etwa $30 \mathrm{~cm}$, die Brechkraft dieses Systems - $36 \mathrm{dptr}$ beträgt, so liegt das im Apparat zur Beobachtung kommende Bild etwa 2,5 cm hinter der Mitte des so weit als möglich nach dem Prüfling 
zu verschobenen Linsensystems, also etwa unter der Spitze des Pfeiles*).

Auf diesen Ort beziehen sich die Werte für die Leseweiten, die mit Hilfe eines das Visier $V$ tragenden Maßstabes gewonnen werden, der in der nach rechts und links (entsprechend der Benutzung des einen oder des anderen Auges) umklappbaren Röhre $R$ verschoben werden kann. Das Visier wird auf die vordere Hornhautkuppe eingestellt, der MaBstab ergibt dann automatisch den Abstand zwischen vorderem Augenhauptpunkt and der virtuellen Sehprobe $X$. Mit Hilfe der auf dem Schneckenrade angegebenen Zahlen lassen sich die Verkleinerungswerte aus einer. Tabelle ablesen. Am Objektträger befindet sich ein kleiner Bügel mit elektrischer Glühlampe.

Der soeben beschriebene Apparat bietet die Möglichkeit zu mancherlei Untersuchungen, von denen die folgenden zunächst ins Auge gefaßt wurden: 1. Vergleich zwischen der Fern m und Nahsehschärfe des ruhenden und des akkommodierenden emmetropischen Auges. 2. Untersuchung von Druckschriften in gewöhnlicher Leseweite, 3. Untersuchung der natürlichen Sehschärfe hochgradiger Myopen.

Die unter 3. genannten Untersuchungen konnte ich bisher noch nicht ausführen; sie sollen von anderer Seite in Angriff genommen werden. Zu 1. und 2. habe ich ausgedehnte Versuchsreihen angestellt, deren Ergebnis hier mitgeteilt werden soll.

\section{Vergleich zwischen Fern- und Nahsehschärfe.}

Exakte Untersuchungen zum Vergleich eines und desselben Auges im ruhenden und akkommodierenden Zustande sind meines Wissens niemals angestellt worden. Wenigstens habe ich in der Literatur nirgends vergleichende zahlenmäBige Angaben über Fern- und Nahsehschärfe finden könner. Zur möglichst genauen Bestimmung der ersteren bediente ich mich des oben erwähnten. Sehprobenschlittens, der das Sehobjekt von 7,6 bis etwa $5 \mathrm{~m}$ anzunähern gestattete, also eine Vergrößerung von $2: 3$ zuließ, sofern man die für die Fernsehprüfung vorgeschriebene Entfernung von $5 \mathrm{~m}$ nicht unterschreiten wollte. Bei den ersten mehr nur orientierenden Versuchsreihen verwendete ich einzeln ausgeschnittene Probebuchstaben aus einer der üblichen

*) Abgeleitet nach der Formel $B=A+D$.

Hier ist $A=-3,33$ dptr

$$
\begin{aligned}
& D=-36,0 \\
& \hline B=-39,33 \mathrm{dpt} r \\
& 1 \\
& B=b=-2,54 \mathrm{~cm} .
\end{aligned}
$$

Die Hauptpunkte des Systems können als zusammenfallend angenommen und in die Mitte des Systems verlegt werden. 
(in Jena lithographierten) Sehprobentafeln. Dieselben erwiesen sich für den vorliegenden Zweck einer möglichst eindeutigen Bestimmung der Fernsehschärfe sehr bald als unbrauchbar, da die verschiedenen Buchstaben und Zahlen sehr stark voneinander abweichende Werte ergaben. Diese Verhältnisse sind ja bekannt und hinreichend oft besprochen worden, besonders ausführlich in letzter Zeit von Gebb und Löhlein ${ }^{12}$ ). Es kam also nur eine Sehprobe ernstlich in Frage, welche ständig dasselbe Objekt zu verwenden gestattete, also etwa der Landoltsche Ring oder die Guillerysche Punktprobe.

Ehe ich auf die Reihen selbst eingehe, möchte ich noch kurz die Versuchsanordnung besprechen.

Der Sehprobenschlitten befand sich in einem halbdunklen Raum, anfänglich in dem Hörsaal der Augenklinik, dessen Jalousien geschlossen waren, später in einem Kellergang. Die Sehproben wurden durch 2 elektrische Glühlampen beleuchtet, deren Helligkeit durch einen einfachen Rheostaten auf optimale, also diejenige Stärke eingestellt werden konnte, bei der der Beobachter am besten lesen zu können meinte; diese Einstellung geschah durch den Assistenten auf Veranlassung der Versuchsperson.

Messungen der Beleuchtungsstärke wurden während der Versuche selbst nicht vorgenommen. Dagegen wurde zweimal nach der üblichen günstigsten Beleuchtung der Sehprobe diese durch ein mattweißes Zeichenpapiex ersetzt und letzteres photometriert. Beim ersten Male ergaben sich mittels zweier verschiedener Messungen 554 bzw. 568, beim zweiten Male 429, im Mittel also etwa 500 Meterkerzen. Da nach Laan und Pickema (zit. nach Löhner ${ }^{4}$ ) die Sehschärfe bereits bei einer Beleuchtung von 30 bis 50 M.K. ihr Maximum erreichen soll, so ist jedenfalls kein Zweifel, daß bei unseren Versuchen die Objekte stets hinreichend stark beleuchtet gewesen sind. Etwa zu große Stärke der Beleuchtung wird durch Adaptation und Pupillenverengerung in weiten Grenzen ausgeglichen; zudem stand es dem Beobachter jederzeit frei, die Beleuchtung verringern zu lassen.

Das Visometer wurde bei den ersten Versuchen bei natürlicher Tagesbeleuchtung benutzt. Dabei war gleichfalls für optimale Beleuchtung gesorgt: Es wurde nur in den Mittagsstunden gearbeitet, der Rücken der Versuchsperson (V. P.) war gegen das Fenster, welches kein direktes Sonnenlicht eintreten ließ, gerichtet. Zur Vermeidung von Reflexen an der Visometerlinse waren die unteren Teile des Fensters abgedeckt, und es wurde ein Pappschirm bzw. ein schwarzes Tuch über den Kopf der V.P. und die Linse des Visometers gedeckt. Später wurde auch an dem Visometer die bereits erwähnte mit Rheostat versehene Beleuchtungsvorrichtung angebracht; die optimale Einstellung konnte hier von der V.P. selbst vorgenommen werden. Die 
Fixation des Kopfes geschah durch eine Kinnstütze mit Stirnhalter, an welchem eine kleine Klappe angebracht war, die das zurzeit nicht untersuchte Auge verdeckte.

Was die Ausführung der Einstellung und Ablesung anbelangt, so ging ieh bei den vorbereitenden mit Buchstaben und Zahlen angestellten Reihen in der Weise vor, daß die Leseprobe aus der größtmöglichen Entfernung von dem Assistenten allmählich herangeschoben wurde, bis sie erkennbar war. Der Beobachter gebot Halt, sowie er glaubte, das Zeichen entziffern zu können. War die Ablesung falsch oder die Erkennbarkeit noch unsicher, so wurde die Probe nach Bedarf weiter genähert. Die Zeichen waren auf Pappstreifen so angebracht, daß sie einzeln hinter einem kleinen, in dem weißen Schirm ausgeschnittenen Fenster eingestellt werden konnten.

Die Versuche mit dem Landoltschen Ring dagegen wurden an dem Sehprobenschlitten größtenteils so vorgenommen, daß dieser zunächst in kleinere, allmählich in größere für je 6 Ablesungen gleichbleibende Entfernungen eingestellt und die Schwelle dann dort angenommen wurde, wo äberwiegend richtige in ubberwiegend falsche Aussagen übergingen. Als Beispiel mögen einige Zahlen aus Reihe 14 dienen:

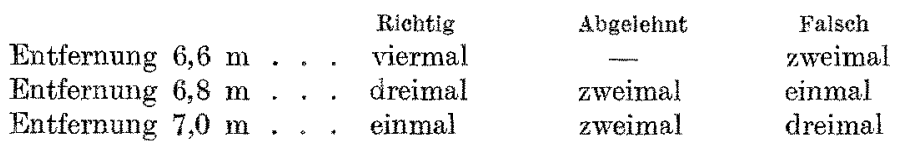

Der Schwellenwert wurde hiernach mit 6,9 $\mathrm{m}$ festgesetzt. Der Landoltsche Ring befand sich bei den Versuchen in der Mitte einer Drehscheibe aus weißem Karton und wurde nur in den 4 Hauptrichtungen eingestellt.

Das eben beschriebene Verfahren der genauen Schwellenermittlung wurde für die eigentlichen Vergleiche der Fern- und Nahsehschärfe wieder aufgegeben; vielmehr benutzte ich auch beim Landoltschen Ring die einfache Methode der Annäherung bis zum richtigen Erkennen. Etwa vorherige falsche Ablesungen blieben dabei unberücksichtigt. Aus den verschiedenen Entfernungen, in denen richtige Ablesung erfolgte, wurde das arithmetische Mittel genommen. Die wenigen, auf diese Weise mit dem Landoltschen Ring von mir selbst mit dem unbewaffneten rechten Auge vorgenommenen Vergleichsablesungen ergaben für die Ferne (Reihe 19): Erkennbarkeit des für $3,33 \mathrm{~m}$ bestimmten Ringes in durchschnittlich $7,34 \mathrm{~m}$, was einer Sehschärfe $=2,2$ entspricht. Am Visometer für die Nähe konnte ich (Reihe 1, 2) dagegen mit demselben Auge bei einem Objektabstand von 250 bzw. 270, durchschnittlich also $260 \mathrm{~mm}$ den für $1,5 \mathrm{~m}$ bestimmten Ring bei Verkleinerungen erkennen, die einer Objektentfernung von 2,42 bzw. $2,28 \mathrm{~m}$ entsprechen würde. Daraus ergab sich mir eine Nahsehschärfe 
von 1,61 bzw. 1,52, durchschnittlich also 1,56. Diese auffallend große Differenz zwischen Fern- $(2,2)$ und Nahsehschärfe $(1,56)$ konnte jedoch nicht ohne weiteres als gültig angenommen werden wegen der Mangelhaftigkeit des von mir bei diesen Versuchen benutzten Sehprobenmaterials. Die Maße eines exakten Landoltschen Ringes nämlich, unter der Voraussetzung, daß sowohl die Ringbreite wie die Lücke unter $1^{\prime}$ erscheinen und daß der äußere Durchmesser dem Fünffachen der Breite entsprechen soll, sind folgende:

\begin{tabular}{|c|c|c|c|}
\hline Erkennungsweite & $\begin{array}{l}\text { Ringbreite } \\
\text { bzw. Liücke }\end{array}$ & $\begin{array}{c}\text { Äußerer } \\
\text { Durchmesser }\end{array}$ & $\begin{array}{c}\text { Innerer } \\
\text { Durchnesser }\end{array}$ \\
\hline & $1,45 \mathrm{~mm}$ & $7,27 \mathrm{~mm}$ & $4,36 \mathrm{~mm}$ \\
\hline & 0,97 & 4,85 , & 2,91 \\
\hline & 0,44, & 2,18, & 1,31, \\
\hline
\end{tabular}

Demgegenüber schwanken die in den "Internationalen Sehproben" (J. F. Bergman n, Wiesbaden, 1913) enthaltenen für $5 \mathrm{~m}$ bestimmten Ringe in folgender erheblichen Weise:

$\begin{array}{lcc}\text { Außendurchmesser zwischen } & 6,5 \text { und }>7,5 \mathrm{~mm} \\ \text { Ringbreite } & " & 1,4 \text { and } 1,8, \\ \text { Lücke } & " & 1,0 \text { and } 1,3,\end{array}$

Die für $3 \frac{1}{3} \mathrm{~m}$ bestimmten Ringe zeigen ähnliche Abweichungen; der von mir an dem Sehprobenschlitten benutzte z. B. maß:

Breite $1,12 \mathrm{~mm}$, Lücke $0,71 \mathrm{~mm}$, Außendurchmesser $5,0 \mathrm{~mm}$, Innendurchmesser $2,76 \mathrm{~mm}$.

Bedeutend genauer sind die Ringe in den Birkhäuserschen Leseproben (Julius Springer, Berlin 1911). Der diesen entnommene für $1,5 \mathrm{~m}$ bestimmte und von mir am Visometer verwendete Ring mißt: Breite 0,44, Lücke 0,41, Außendurchmesser 2,24, Innendurchmesser 1,32 mm, stimmt also außerordentlich gut mit den vorschriftsmäßigen Werten überein.

Um den Vergleich zwischen Fern- und Nahsehschärfe mittels des internationalen Landoltschen Ringes genau festzulegen, müßten deshalb für Schlitten und Visometer von ein und demselben sorgfältig hergestellten Objekt auf photographischem Wege verschieden starke Verkleinerungen hergestellt werden, wie es bei der nunmehr zu beschreibenden neuen Sehprobe geschehen ist.

Bei allem Beifall, den der Landoltsche Ring gefunden hat, ist doch öfters betont worden, daß er ein ziemlich kompliziertes Objekt darstellt, so daß die Vorgänge, die beim Erkennen desselben stattfinden, gar nicht ganz einfach zu analysieren sind. Landolt ${ }^{13}$ ) selbst hat außerdem darauf hingewiesen, daß der Ring für Astigmatiker in den verschiedenen Richtungen verschieden schwer zu erkennen ist, und hält dies für einen Vorzug seiner Probe. Es mag dies für praktische Untersuchungen allenfalls zutreffen, für Versuche entsprechend den vorliegenden sehe 
ich darin einen Mangel des Landoltschen Ringes. Denn man kann sich meines Erachtens nicht auf den Standpunkt stellen: Für physiologische Untersuchungen. kämen überhaupt nur völlig astigmatismusfreie Augen in Frage. Denn etwas völlig Fehlerfreies gibt es nicht in der Natur, Meine eigene Sehschärfe beträgt, wie bereits erwähnt, zwischen 1,5 und 2,0, und trotzdem sind meine Augen nicht ganz frei von Astigmatismus: Ein Zylinder von $+0,25$ dptr mit etwa vertikaler Achse ergibt vor meinem Auge zwar noch keine ohne weiteres nachweisbare Steigerung der Sehschärfe, jedoch subjektiv eine deutlich erhöhte Brillanz der Sehobjekte, Drehung dieses Zylinders um $90^{\circ}$ rogar schon merkliche Verschlechterung.

Dem Landoltschen Ringe gegenüber würde die Guillerysche Punktprobe an Einfachheit und ständiger Gleichwertigkeit nichts zn wünschen übrig lassen. Ich habe mich trotzdem nicht entschließen können, die weiteren Versuche mit dieser Probe anzustellen, weil es mir immer als eine Schwierigkeit erschienen ist, daß dabei das Auge, so lange es



a

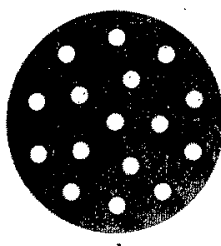

b den Punkt noch nicht erkennt, nichts zum Fixieren hat, sondern beständig den Raum absuchen muB. Diesen Mangel habe ich geglaubt auf folgende Weise überwinden zu können: Als Sehprobe verwendete ich nicht einen einfachen Punkt, sondern einen geschlossenen schwarzen

Ring von gleicher Öffnung wie Breite, welcher also anßen den dreifachen Durchmesser wie innen hat und als Ganzes um ein Vielfaches früher erkannt wird als seine runde Lichtung (Abb. $5 \mathrm{a}$ ). Letztere stellt das eigentliche punktförmige Sehobjekt dar. Das Erkennen besteht darin, daß beim Annähern die zunächst nur als Flock erscheinende Probe als Ring mit weißem Innenraum wahrnehmbar wird. Objektiven Anhalt für das Erkennen des Ringes gibt der Vergleich mit einem Verwechslungsobjekt von gleich großer, kreisförmiger Außenbegrenzung, in welchem aber statt der beschriebenen weißen Innenfläche 16 kleine gleichmäBig verteilte Kreischen ausgespart wurden, die zusammengenommen den gleichen Flächeninhalt haben; denn ihr Durchmesser beträgt 1/4 des Durchmessers der Innenfläche des Ringobjektes ( $s_{\text {. }} \mathrm{Abb} .5 \mathrm{~b}$ ). Der gesamte Schwarzwert beider. Vergleichsobjekte ist also gleich und sie erscheinen in der Tat auch, solange die Ringform unkenntlich ist, als zwei ganz gleichartige Flecken. Diese ,Fleckprobe" behält thren Charakter bei allen Versuchen unverändert bei, denn ihre kleinen weißen Punkte würden ja erst bei Annäherung anf etwa den vierten Teil sichtbar werden.

Wie sich meine Proben hinsichtlich ihrer Erkennbarkeit zu Guillerys Punkten verhalten - ob sie bei gleicher Größe schwerer oder leichter 
erkennbar sind - habe ich nicht untersucht. Es ist dies ja auch für die vorliegenden Versuche ohne Bedeutung. Daß bei mir im Gegensatz zu Guiller y nicht ein schwarzer, sondern ein weißer Punkt als Probeobjekt dient, halte ich, wenn nicht für belanglos, so eher für einen Vorteil als einen Nachteil, denn die Helligkeit ist für unser Auge doch das positiv Wahrnehmbare. Andererseits verhehle ich mir nicht, daß durch den schwarzen Ring, von dem mein Probepunkt umgeben ist, meine Versuchsbedingungen gegenüber denen von $G$ uillery in bezug auf Kontrasterscheinungen usw. wesentlich verwickelter werden. Dies scheint mir aber nur für Untersuchungen über die Natur des Sehens selbst von Bedeutung, nicht für die vorliegenden vergleichenden Sehschärfeprüfungen.

Als Normalgröße der Ringfleckprobe habe ich angenommen, daß das weiße Scheibchen unter einem Gesichtswinkel von $I^{\prime}$-erscheinen soll, entsprechend der Snellenschen Bedingung; der Außendurehmesser beträgt, wie erwähnt, das Dreifache des inneren. Eine Probe von dieser Größe ist, wie die nach-


folgenden Zahlen auch zeigen werden, schwerer zu erkemnen als ein Landoltscher Ring von vorgeschriebener Größe und erheblich schwerer als Buchstaben nach dem Snellenschen Prinzip.

Die beiden Objekte wurden von mir selbst in beträchtlicher Größe mit Zirkel und Tusehe hergestellt und von dem Mitarbeiter der Firma Zeiss; Herrn Dr. Gu und-

lach, in mustergültiger Weise photographisch verkleinert; mir standen dann sehr exakte Objekte in 3 verschiedenen Größen zur Verfügung, von denen die mittlere nur zu Vorversuchen Verwendung fand.

Die mikroskopische Messung des großen, an dem Schlitien benutzten Objektes ergab: Ringbreite 1,45, lichte Weite 1,48, Außendurchmesser $4,38 \mathrm{~mm}$; es mußte danach für eine Sehschärfe $=1$ in $5,02 \mathrm{~m}$ erkannt werden. Die Maße des kleinen für das Visometer bestimmten Objektes waren: Ringbreite 0,63, lichte Weite 0,65, Außendurchmesser $1,91 \mathrm{~mm}$. Sehschärfe $=1$ galt demnach für $2,19 \mathrm{~m}$.

Bei den Versuchen nun wurde nicht etwa nur 1 Ring und 1 Fleck zum Vergleiche geboten, sondern es wurden auf einer Drehscheibe in Kreisform 8 Objekte angebracht, und zwar 7 Flecken und 1 Ring. Es waren also 8 verschiedene Einstellungen möghich, wodureh ein Raten ausgeschlossen erschien. Die einzelnen Objekte standen etwa so weit auseinander, daß in jedem Zwischenraum noch 2 weitere Platz gefunden hätten (s. Abb.). Eine gegenseitige Beeinflussung der Objekte 
fand dabei nicht mehr statt und doch waren nur kleine Blickschwankungen nötig, um den ganzen Kreis zu durchsuchen. Die Beleuchtungsverhältnisse an dem Sehprobenschliten und am Visometer waren unverändert wie bei den Versuchen mit dem Landoltschen Ring, ebenso das Verfahren der allmählichen Ammäherung bis zum Kenntlichwerden.

Zur möglichst genauen Bestimmung der Fernsehschärfe meines rechten Auges wurden an drei kurz aufeinanderfolgenden Tagen je 20 Ablesungen an dem Sehprobenschlitten vorgenommen. Die Ergebnisse waren folgende:

Erkennungseite:

Reine

1. (4. III. 1914)

2. (5. III. 1914)

3. (7. III. 1914) Gropter Wert Kleinster Wert Durch

$\left.\begin{array}{llll}6,45 & 5,25 & 5,94 & 1,18 \\ 7,10 & 5,90 & 6,46 & 1,29 \\ 7,40 & 6,20 & 6,94 & 1,38\end{array}\right\} \mathbf{1 , 2 8}$

Die entsprechenden Versuche mit demselben Ange am Visometer wurden teils an demselben, teilis an dazwischenliegenden Tagen mit folgendem Ergebnis angestellt:

$\begin{array}{ccccc}\text { Verkleinerung entsprechend einer } & \text { Erkennungsweite von: } \\ \text { Reihe } & \text { Gröbter Wert } & \text { Kleinster Wert } & \text { Durchschnitt } & \text { Nahsehsehärfe } \\ \text { 1. (3. III. 1914) } & 2,52 & 1,84 & 2,20 & 1,0 \\ \text { 2. (4. III. 1914) } & 3,02 & 2,04 & 2,59 & 1,18 \\ \text { 3. (6. III. 1914) } & 3,20 & 2,20 & 2,66 & \mathbf{1 , 1 3}\end{array}$

Die Nahsehschärfe verhielt sich also zur Fernsehschärfe wie $1,13: 1,28$ oder wie $0,88: 1,0$.

Es kann wohl nicht bestritten werden, daB die mitgeteilten sowohl für den Sehprobenschlitten wie für das Visometer ständig anwachsenden Sehschärfezahlen mindestens teilweise auf immer noch zunehmende Ùbung zurückzuführen sind. Zur Gewinnung absolut gültiger Werte hätten also noch mehr Versuche, bis zur Erreichung einer größeren GleichmäBìgkeit, angestellt werden müssen. Doch glaube ich aus den mitgeteilten Reihen schon mit Sicherheit entnehmen zu können, daß die Fernsehschärfe der Nahsehschärfe in allen Fällen überlegen ist.

Die Leseweite betrug bei den Visometerversuchen in Reihe $1=353,0$, Reihe $2=341,5$, Reihe $3=345,5$, durchschnittlich $\mathbf{3 4 6 , 5} \mathbf{m m}$, die Akkommodation war also noch verhältnismäßig wenig angespannt. Weitere Versuche mit allmählich verringertem Leseabstand wurden vor dem Kriege von mir leider noch nicht angestellt; z. Z. steht mir das Visometer nicht mehr zur Verfügung. Es wäre aber sehr interessant zu erfahren, ob bei größerer Annäherung der Objekte, wie es den Anschein hat, die natürliche Sehschärfe tatsächlich noch weiter sinkt. Besonders lohnend wäre in diesem Zusammenhange ein Vergleich mit den Sehschärfeverhältnissen der Hyperopen. welche unkorrigiert ja 
schon für die Ferne akkommodieren müssen, also dabei schon in gleicher Lage sind wie ein Emmetrop beim Nahesehen.

Mehrere Versuchsreihen, die Kollege Erggelet die Freundlichkeit hatte für mich auszuführen, bestätigten mir das Ergebnis, daß die Nahsehschärfe erheblich geringer ist als die Fernsehschärfe. Diese Versuche wurden unter genau den gleichen Bedingungen wie bei mir (in jeder Reihe 20 Ablesungen) mit dem rechten Auge vorgenommen, und ergaben für die Ferne:

Erkennungsweite:

\section{Reihe}

1. (18. III. 1914)

2. (24. III. 1914)
Gröbter Wert Kleinster Wert D

$\left.\begin{array}{lll}5,15 & 4,0 & 4,48 \\ 5,25 & 4,0 & 4,52\end{array}\right\} \quad 0,90$

und für die, Nähe:

Verkleinerung entsprechend einer Erkennungsweite von: Reihe

1. (19. III. 1914)

2. (21. III. 1914) Größter Wert Kleinster Wert Durchschnitt Nahsebschärie

$\left.\begin{array}{lll}1,67 & 1,15 & 1,44 \\ 1,57 & 1,20 & 1,43\end{array}\right\} \quad \mathbf{0 , 6 6}$

Diese Reihenpaare zeigen eine bemerkenswert gute Uthereinstimmung.

Bei den Visometerreihen betrug die Leseweite 284,0 bzw. 272,0; durchschnittlich 278,0 mm. Das Verhältnis zwischen Nah- und Fernsehschärfe ist $0,66: 0,90$ oder $0,73: 1,0$, ist also für die Nahsehsehärfe noch wesentlich ungünstiger als bei mir. Welche Rolle dabei die Verschiedenheit der Versuchsperson und welche die größere Objekt annäherung spielt, müßte, wie gesagt, durch. weitere Versuche planmäßig festgestellt werden.

\section{Vergleichung von Druckschriften.}

Ich komme jetzt zu dem an zweiter Stelle erwähnten. Anwendungsgebiete des Visometers, der Untersuchung von Druckschriften in gewöhnlicher Leseweite. Es handelt sich hierbei um ein Problem, welches kurz durch die Streitfrage: „Antiqua oder Fraktur?" ausgedrückt werden kann und welches in den letzten Zeiten hauptsächlich von Psychologen bearbeitet worden ist. Dieselben haben sich dabei im wesentlichen dreier Arbeitsmethoden bedient: 1. Der tachistokopischen, wobei eine Schriftprobe (Einzelbuchstabe oder Wort) nur momentan dargeboten wird [Erdmann und Dodge $\left.{ }^{14}\right), Z_{\text {eitler }}{ }^{15}$ ), Schumann $n^{16}$ ), Messmer ${ }^{17}$ ) u. a. ], 2. Der Prüfung des indirekten Sehens [Erdmann und Dodge, Kirschmann ${ }^{18}$, wobei von der Voraussetzung ausgegangen wird, daß auch beim gewöhnlichen Lesen nur vereinzelte Punkte eines dargebotenen Textes genau fixiert, das meiste aber indirekt erkannt wird, und 3. der nystagmographischen Methode [Schackwitz ${ }^{19}$ ), Lobsien $\left.{ }^{20}\right)$ ], welche darin besteht, daß mittels einer Registriervorrichtung die Zahl der Rucke, die das Auge beim Lesen macht, 
aufgezeichnet wird. Ich selbst bin der Meinung, daB alle diese Verfahren, besonders die beiden letzteren, mehr Beziehungen zurn natürlichen Lesevorgang haben, als die Untersuchung der Erkennbarkeit einer ruhenden Sohriftprobe im zentralen Sehen, und daß deshalb die Entscheidung in der Schriftfrage von den Psychologen gefällt werden muB (sie scheint, soviel ich sehen kann, sich der Fraktur zuzuneigen!); aber trotzdem schien es mir nicht unnütz, die jetzt mitzuteilenden Versuche mit dem Visometer auszuführen. Denn der Apparat brachte zwei große Vorteile: 1. Das Objekt in der gewöhnlichen Leseweite darbieten und ohne diese $\mathrm{zu}$ ändern es beliebig vergrößern and verkleinern zu können, 2. die Möglichkeit der Verwendung üblicher, für den gewöhnlichen Buchdruck bestimmter Schriftzeichen. Ohne das Visometer war eine Prüfung in der üblichen Leseweite mit stetiger Größenänderung: auf keine Weise möglich, und wenn man überhaupt das Objekt allmählich in seiner Größe wachsen lassen wollte, mußte man entweder Schiftzeichen von ungewöhnlichen Maßen anwenden (die in der Regel in ihren Proportionen mit den gewöhnlichen Druckschriften nicht genau übereinstimmen) oder aber auf die eigentlich selbstverständliche Forderung verzichten, daßs während der Untersuchung der Akkommodationszustand des Versuchsaugos nicht merklich verändert werden darf. In diesen Beziehungen also war ich in Vorteil gegenüber früheren Untersuchern, die schon Sohriftarten nach Art einer Sehschärfeprifung verglichen haben, und glaube deshalb, daB meine Ergebnisse eine willkommene Ergänzung der erwähnten anderen Leseforschungen bilden können. Bei früheren Sohriftvergleichungen im direkten Sehen. scheint sich im allgemeinen eine Utberlegenheit der Antiqua ergeben zu haben. Eine Eingabe des, ,Allgemeinen Vereins für Altschrift", welche im Februar 1911 dem Reichstag vorgelegt worden ist21), behauptet, für gleich große Antiqua and Fraktur betrügen die Entfernungen

für das Entziffern $143 \mathrm{~cm}$ (Ant.) und $115 \mathrm{~cm}$ (Frakt.), für das geläufige Lesen $115 \mathrm{~cm}$ (Ant.) und $91 \mathrm{~cm}$ (Frakt.).

Das würde ein durchschnittliches Verhältnis der Leserlichkeit der Fraktur zu der der Antiqua von 1,0:1,25 bedeuten. Die Eingabe stützt sich dabei auf Untersuchungen besonders von F. Soen necken.

Zur Vergleichung der Druckschriften waren mir auf Anregung des Herrn Professors von Rohr in liebenswürdiger Weise von der Firma. B. G. Teub ner, Leipzig, Versuchsdrucke zur Verfügung gestellt worden, welche aus einer Seite eines staatswissenschaftlichen Textes und aus den einzeln gedruckten großen und kleinen Buchstaben bestand. Die Probe war insofern sehr glücklich gewählt, als sie neben einer großen Anzahl kurzer und landläufiger Worte auch, reichlich solche von großer und mittlerer Länge enthielt, darunter auch Worte, die nur sehr selten 
vorkommen. Zum Vergleich diente dreimal derselbe Textabschnitt: in gewöhnlicher Zeitungsfraktur, in gewöhnlicher Antiqua und in Offenbacher Schwabacher; alle 3 Schriften waren von genau gleicher Höhe (Borgis), aber entsprechend dem Charakter der Schriften nicht genau gleicher Breite; in welcher Weise diese letztere Ungleichheit rechnerisch berücksichtigt wurde, soll bei der Besprechung der Ergebnisse erörtert werden.

Die Ablesungen wurden sämtlich von mir selbst mit dem rechten Auge vorgenommen, in gleicher Weise wie bei den Sehschärfebestimmungen am Visometer: allmähliche Vergrößerung bis zur Erkennbarkeit; das Einstellrad wurde dabei von mir selbst bedient. Künstliche günstigste Beleuchtung, wie bisher. Als Gehilfe diente während sämtlicher Versuche die gleiche Person, welche den Buchstaben bzw. das Wortbild in ein kleines Rähmchen einzustecken und nach erfolgter Ablesung die Vergrößerungszahl aufzuschreiben hatte. Die Worte waren von mir selbst mehrere Tage vorher ausgeschnitten und auf kleine Kartonstreifen geklebt worden; ich hatte während der Versuche nur noch eine ganz schwache Erinnerung, welche Worte etwa in der Reihe enthalten waren. Die 3 Schriftarten wurden bei einem Teil der Reihen in regelmäßigem Wechsel, bəi einem anderen Teil völlig unregelmäßig durcheinander dargeboten; vielfach konnte von von mir bei den Ablesungen überhaupt nicht festgestellt werden, welche Schriftart vorlag. Alle die Schriftablesungen wurden im Laufe von etwa fünf aufeinanderfolgenden Wochen (Januar/Februar 1914) vorgenommen, zu einer Zeit, als durch die vorhergehenden Untersuchungen schon eine erhebliche Sicherheit im Beobachten erreicht war. Die ZahI der Ablesungen an einem Tage betrug nicht mehr als etwa 50 . Hierdurch scheint mir eine erhebliche Gewähr für Gleichmäßigkeit und Zuverlässigkeit gegeben zu sein.

Zuerst wurden die Einzelbuchstaben untersucht mit Hilfe von 5 Beobachtungsreihen, deren jede aus etwa 150 Einzelbeobachtungen bestand, da sich jede Reihe aus je etwa 25 Groß- und 25 Kleinbuchstaben der 3 verglichenen Schriftarten zusammensetzte. Kleine Abweichungen von der Zahl 25 erklären sich dadurch, daß z. B. bei den Antiqua- und Schwabacher-Majuskeln außer I auch ein $J$ vertreten war und daß unter den Kleinbuchstaben der Fraktur und Schwabacher außer dem runden ein langes s und zudem bei allen 3 Schriftarten ein $\beta$ vorkommt.

Die Ergebnisse werden ausgedrückt durch die aus Leseabstand und Verkleinerung berechnete Zahl (in Meter), die angibt, in welcher Entfernung das nicht verkleinerte Objekt erkannt worden wäre.

Wenn es mir auch bei der ganzen Untersuchung nur auf die Gewinnung zuverlässiger Durchschnittszahlen ankam, so möehte ich doch 
des Interesses halber auch die für jeden großen und kleinen Finzelbuchstaben aus der Zusammenstellung der 5 Reihen gewonnene Finzelzahl hier mitteilen. Ich beschränke mich dabei der Ubersichtlichkeit halber auf die Buchstaben, die in allen 3 Alphabeten zugleich vorkommen.

\begin{tabular}{|c|c|c|c|c|c|c|}
\hline & \multicolumn{2}{|c|}{ Fraktur } & \multicolumn{2}{|c|}{ Antiqua } & \multicolumn{2}{|c|}{ Schwabacher } \\
\hline & Groß & Klein & Groß & Klein & Grob & Klein \\
\hline A & 1,79 & 1,44 & 2,20 & 1,45 & 2,00 & 1,70 \\
\hline$B$ & 1,74 & 1.70 & 1,54 & 1,75 & 1,79 & 1,79 \\
\hline $\mathrm{C}$ & 1,87 & 1,63 & 2,06 & 1,53 & 2,22 & 1,69 \\
\hline $\mathrm{D}$ & 1,96 & 1,73 & 2,08 & 2,05 & 1,91 & 1,86 \\
\hline $\mathbf{E}$ & 1,75 & 1,42 & 2,00 & 1,52 & 1,88 & 1,47 \\
\hline$F$ & 1,89 & 1,59 & 1,77 & 1,16 & 2,19 & 1,47 \\
\hline G & 1,80 & 1,52 & 1,70 & 1,51 & 1,79 & 1,66 \\
\hline H & 1,98 & 1,85 & 1,96 & 1,85 & 2,10 & 2,15 \\
\hline$I$ & 1,75 & 1,62 & 1,64 & 1,44 & 2,28 & 1,31 \\
\hline $\mathbf{K}$ & 1,64 & 1,57 & 1,91 & 1,58 & 1,86 & 1,65 \\
\hline$L$ & 1,64 & 1,70 & 2,44 & 1,51 & 1,76 & 1,71 \\
\hline$M$ & 1,91 & 1,56 & 2,06 & 1,83 & 2,24 & 1,88 \\
\hline $\mathrm{N}$ & 1,57 & 1,55 & 2,10 & 1,39 & 1,64 & 1,55 \\
\hline 0 & 2,03 & 1,34 & 2,49 & 1,83 & 1,87 & 1,71 \\
\hline$P$ & 1,72 & 1,92 & 2,01 & 1,98 & 2,52 & 2,02 \\
\hline $\mathbb{Q}$ & 1,94 & 1,62 & 1,76 & 1,92 & 1,98 & 1,85 \\
\hline$R$ & 1,51 & 1,72 & 1,76 & 1,47 & 1,84 & 1,69 \\
\hline$\$$ & 1,97 & 1,49 & 1,81 & 1,36 & 1,93 & 1,65 \\
\hline $\mathbf{T}$ & 1,96 & 1,38 & 2,17 & 1,26 & 2,00 & 1,48 \\
\hline $\mathrm{U}$ & 2,26 & 1,45 & 1,93 & 1,73 & 2,20 & 1,72 \\
\hline$V$ & 1,37 & 1,58 & 2,31 & 1,74 & 2,16 & 1,51 \\
\hline$W$ & 1,81 & 1,87 & 2,38 & 1,84 & 2,22 & 1,81 \\
\hline$X$ & 1,91 & 1,62 & 2,08 & 1,52 & 1,96 & 1,60 \\
\hline$Y$ & 1,77 & 1,50 & 2,34 & 1,73 & 2,03 & 1,65 \\
\hline$Z$ & 2,03 & 1,53 & 2,06 & 1,47 & 1,90 & 1,76 \\
\hline
\end{tabular}

Es liegt mir natürlich fern zu glauben, daß diesen Einzelzahlen eine maßgebliche Bedeutung zukommen könnte; dazu würden wesentlich mehr als 5 Ablesungen jedes einzelnen Buchstaben erforderlich sein. Ich habe diese Werte nur gewissermaßen als die Unterlage mitgeteilt, aus dem die folgenden Verhältniszahlen gewonnen wurden. Diese lauten:

\begin{tabular}{|c|c|}
\hline Fraktur-Großbuchstaben & $1,82 \mathrm{~m}$ \\
\hline Kleimbuchstaben & $1,59 \mathrm{~m}$ \\
\hline $\begin{array}{r}\text { Antiqua-Großbuchstaben } \\
\text { " Kleinbuchstaben }\end{array}$ & $\left.\begin{array}{ll}2,01 \mathrm{~m} \\
1,59 \mathrm{~m}\end{array}\right\} 1,80 \mathrm{~m}$ \\
\hline $\begin{array}{r}\text { ahwa bacher-Großbuchstaben } \\
\text { Kleinbuchstaben }\end{array}$ & $\begin{array}{l}2,02 \mathrm{~m} \\
1,69 \mathrm{~m}\end{array}$ \\
\hline
\end{tabular}

Hier ist es nun nötig, die oben bereits erwähnten ungleichen Breitenverhältnisse der 3 Schriftarten in ihrem Einfluß auf die Lesbarkeit zu besprechen. Jeder, der sich bisher mit Druckschriften befaßte, 
hat es ohne weiteres für selbstverständlich gehalten, daß nur gleiche Schriftgrade, bei denen also die Buchstaben genau gleiche Höhe haben, miteinander verglichen werden können. Über die Breite ist man dagegen im allgemeinen mit Stillschweigen hinweggegangen, indem deren Verschiedenheit gewissermaßen zu den unvermeidlichen Eigentümlichkeiten einer Schrift gehöre, die weiter zu berücksichtigen man nicht in der Lage wäre. Noch auf der Leipziger Bugra im Jahre 1914. begegnete mir eine Versuchsanordnung, die dazu bestimmt war, die angebliche Überlegenheit der Antiqua einem Laienpublikum vorzuführen; auch hier lief, wie gewöhnlich, die angewendete Fraktur erbeblich schmäler als die damit verglichene Antiqua und niemand dachte daran, diese Verschiedenheit etwa durch eine Zulage in der Höhe auszugleichen. Es ist abər gar nicht einzusehen, weshalb die Höhendimension wesentlich, die Breite aber bedeutungslos sein soll. Vielmehr darf die eine ebenso wenig vernachlässigt werden wie die andere; ausschlaggebend ist das Produkt aus beiden, die Fläche, und gleich gute Lesbarkeit zweier entsprechender Buchstaben von verschiedener Sehriftart liegt dann vor, wenn beide bei gleicher scheinbarer Flächengröße erkannt werden*). Die scheinbare Flächengröße eines beliebigen Gegenstandes aber ist umgekehrt proportional dem Quadrate seiner Entfernung vom Auge, denn bei den kleinen hier in Frage kommenden Winkeln können diese, welche das Maß der scheinbaren Größe darstellen, mit ihren Tangentenwerten vertauscht werden. Gleiche scheinbare Flächengröße zweier Buchstaben $h a$ und $h b$ von gleicher Höhe $h$, aber ungleicher Breite $a$ und $b$ wird also dann bestehen, wenn die Flächen der beiden Buchstaben im umgekehrten Verhältnis zueinanderstehen wie die Quadrate ihre Entfernung vom Auge.

Nennen wir diese Entfernungen $r_{a}$ und $r_{b}$, so gilt also:

$$
\begin{aligned}
& h a: h b=r_{b}^{2}: r_{a}^{2}, \\
& r_{t}^{2}=r_{b}^{2} \cdot \frac{b}{a}, \\
& r_{a}=r_{b} \cdot \sqrt{\frac{b}{a}} .
\end{aligned}
$$

$\frac{b}{a}$ ist das Verhältnis der Breiten der verglichenen Buchstaben. Man

*) Sowohl für das Erkennen beim indirekten Sehen, welches beim wirklichen Iesen ja eine so große Rolle spielt, wie auch mit Rücksicht auf Zahl und GröBe der beim Lesen auftretenden Augenrucke kann die größere oder geringere Breite einer Schrift noch eine andere und unter Umständen bedeutungsvollere Rolle spielen, als wie bei der einfachen Flächenberechnung zum Ausdruck kommt. Für die vorliegenden Untersuchungen indes, bei welchen zentrales Sehen und ruhendes Auge gegeben war, muß ich mich darauf beschränken, beiden Dimensionen gleiche Bedeutung beizumessen. 
findet also die Entfernung $r_{a}$, in der der Buchstabe $h a$ in gleicher Flächengröße erscheint wie der in $r_{b}$ befindliche Buchstabe $h b$, wenn man $r_{b}$ mit der Quadratwurzel aus $\frac{b}{a}$ (dem Breitenverhältnis) multipliziert.

Das soeben Auseinandergesetzte kann man sich auch folgendermaBen klarmachen: 2 Druckschriften sind nur dann als gleich groß zu betrachten, wenn von beiden die gleiche Menge Text auf eine Seite geht; es kommt für den Vergleich also auf ihr Flächen-, nicht auf ihr Höhenverhältnis allein an. Stimmt ein Vergleichspaar von Buchstaben demnach in einer Dimension (der Höhe) überein, so ist der in der zweiten Dimension (der Breite) weniger ausgedehnte Buchstabe dem anderen bei gleicher Leseweite dem Breitenverhältnis entsprechend überlegen; die Lesbarkeit verhält sich umgekehrt wie das Breitenverhältnis. Wie steht es aber nun mit der Lesbarkeit zweier flächengleicher Buchstaben, die in ungleicher Entfernung erkannt werden? Offenbar verhält sich diese wie das Quadrat der Abstände, denn die scheinbare Flächengröße vermindert sich ja mit dem Quadrate der Entfernung. Genau genommen müßten demnach alle Leseweiten, die in vorstehendem als $\mathrm{Ma} B$ verwendet worden sind, ins Quadrat erhoben werden, um die Unterschiede der Lesbarkeit zum Ausdruck zu bringen. Ganz entsprechend hat auch Guillery ${ }^{22}$ ) die zweifellos völlig begründete Forderung aufgestellt, daß die Sehschärfe nicht den Leseweiten, sondern ihrem Quadrate entsprechend anzusetzen sei; denn ein Auge, welches nach der üblichen Bezeichnung die doppelte Sehschärfe hat, wie ein anderes, vermöge tatsächlich viermal so kleine Gegenstände zu erkennen.

Es genügt jedoch, dies zu wissen; in praxi kann man dann ebensogut die einfache Leseweite als Maß der Sehschärfe verwenden. Und dasselbe gilt für die Lesbar keit; auch sie kann man ebensogut einfach mit der Leseweite steigen und fallen lassen; nur dart man dann zum Ausgleich von Abweichungen in nur einer Dimension (gleicho Höhe, ungleiche Breite) nicht mehr das Breitenverhältnis selbst, sondern nur dessen Quadratwurzel verwenden; denn man gibt ja in Wahrheit auch nur die Quadratwurzeln der Lesbarkeit.

Um wieder zu den untersuchten Schriften zurückzukommen, so benötigte der verwendete Abschnitt, welcher in Fraktur $3092 \mathrm{~mm}$ lang war, in Antiqua, $3313 \mathrm{~mm}$ und in Schwabacher $3236 \mathrm{~mm}$. Das Breitenverbältnis ergibt sich hiemach = Fraktur: Antiqua : Schwabacher $=1,0: 1,071: 1,047$. Die zu den Reduktionen zu verwendenden Quadratwurzeln daraus lauten $1,0: 1,035: 1,023$.

Der Übersichtlichkeit halber wiederhole ich jetzt die vorhin mitgeteilten Resultate noch einmal und setze das Ergebnis der Umrechnung daneben. 


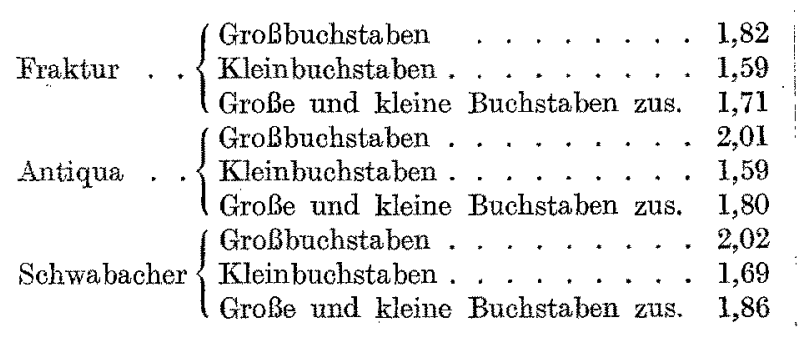

Reduktion dureh 1,0 bzw. 1,035 brw. 1,023 ergibt:

1,82
1,59
1,71
1,94
1,54
1,74
1,97
1,65
1,82

Das Verhältnis der 3 Schriftarten zueinander ist danach folgendes, Fraktur $=1$ gesetzt:

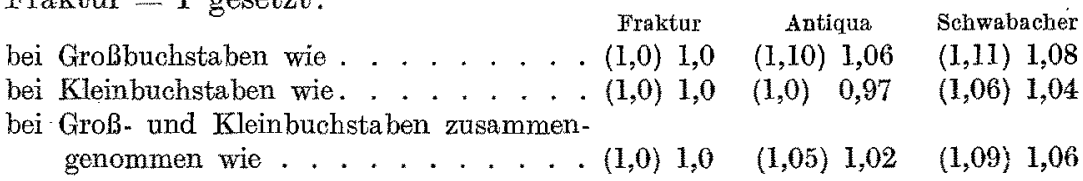

In dieser Zusammenstellung stehen die Zahlen, wie sie sich ursprünglich ergeben, in Klammern vor den umgerechneten.

Utber die Bedeutung dieser Ergebnisse wäre folgendes zu sagen:

In den Zahlen für die Großbuchstaben kommt die allgemein bekannte und von Gegnern der Fraktur viel betonte Tatsache deutlich zum Ausdruck, daß die Fraktur-Großbuchstaben für sich allein schwer zu erkennen sind. Doch ist es hier nicht die Antiqua, welche am allerbesten abschneidet, sondem die Schwabacherschrift erscheint dieser noch überlegen, selbst wenn man zugibt, daß es zweifelhaft sein kann, ob für die Großbuchstaben eine Umrechnung in der hier vorgenommenen Weise zulässig ist: denn die größere oder geringere Breite einer Schriftart beruht wohl im wesentlichen auf den Kleinbuchstaben.

In dem Verhältnis der Kleinbuchstaben schneidet die Antíqua am schlechtesten $a b$. Daneben besteht eine auffallende und auf den ersten Blick nicht recht erklärbare Verschiedenheit zwischen Fraktur und Schwabacher. Wenn man bedenkt, daß die beiden Schriftarten in den Formen der Minuskeln vollkommen übereinstimmen, so läßt sich dies wohl nur so deuten, daß hier gerade der etwas breitere Schnitt der Schwabacher für den einzeln dargebotenen Buchstaben eine unverhältnismäBig bessere Lesbarkeit ergibt. Auf Grund dieser völligen Übereinstimmung in den Formen erscheint es mir erlaubt, zum Zweck der Gegenüberstellung die Zahlen von Fraktur und Schwabacher zusammenzuziehen. Man käme dabei auf einen Mittelwert von 1,02, demgegenüber die Antiqua $(0,97)$ um 5\% unterlegen erseheint.

Auf die Zahlen, in denen Groß- und Kleinbuchstaben zusammengefaßt werden, kann ich keinen besonderen Wert legen; denn eine Mischung von Buchstaben, bei der Majuskeln in ebenso großer Zahl auftreten wie Minuskeln, kommt ja praktisch nicht vor. Es handelt 
sich eigentlich also nur um eine ganz abstrakte Zusammenziehung der vorher besprochenen Resultate, wobei die charakteristischen Ergebnisse verwischt werden. Schwabacher, welche sowohl in den großen wie in den kleinen Einzelbuchstaben gute Leserlichkeit gezeigt hatte, steht hier weitaus an der Spitze, während. Fraktur und Antiqua einander nahe kommen, erstere noch etwas schlechter als letztere, entsprechend dem Umstande, daB die Fraktur-GroBbuchstaben bei der Einzelprüfung eine besonders große Unterlegenheit gezeigt hatten.

Damit verlasse ich die Einzelbuchstaben und wende mich der Prüfung von Wortbildern zu. Da in einem Schriftstìck nur ausnahmsweise einzelne Buchstaben auftreten und ein zu einem Worte zusammengestelltes Buchstabenkonglomerat beim geläufigen Lesen nicht in seinen Einzelheiten, sondern nur in seiner charakteristischen Gesamtform aufgefaßt wird, so kommt der Wortbildervergleichung für die Beurteilung einer Sohrift eine viel größere Bedeutung zu als den für Finzelbuchstaben gewonnenen Zahlen.

Ich habe im ganzen 6 Wortbilder-Beobachtungsreihen angestellt, und zwar 2 , in denen die 3 Schriftarten gleichzeitig, 2 weitere, in denen Fraktur und Antiqua, und schließlich noch 2, in denen Antiqua und Schwabacher miteinander verglichen wurden. In jeder Reihe wurden von jeder beteiligten Schriftart 50 Worte gelesen, so daß also die beiden ersten Reihen je 150, die 4 anderen je 100 Einzelablesungen umfaBten. Durch eine gute Mischung der Kärtchen war dafür gesorgt, daB die verschiedenen Worte, welche, wie bereits erwähnt, in gleicher Weise in allen 3 Schriftarten vorkamen, bald in der einen, bald in der anderen, bald in der dritten Schrift zuerst eingestellt wurden. Dadurch, daß ich in den 4 letzten Reihen nur Antiqua und eine der Bruchschriften gegenüberstellte, wollte ich mich vor einer Benachteiligung der Antiqua schützen. Denn immerhin sind die Wortbilder der Fraktur und Schwabacher einander etwas ähnlicher als denen der Antiqua, so daß vielleicht ein Wort, welches schon in einer der Bruchschriften geboten war, in der zweiten besonders leicht wiedererkannt wurde. Daß dieser Umstand in der Tat keine Rolle gespielt hat, ergibt sich ans der völligenUtbereinstimmung der Ergebnisse der späteren gegenüber denen der ersten Reihen.

Es sind num also im ganzen von Antiqua 300, von Fraktur und Schwabacher nur je 200 Ablesungen gemacht worden. Die Ergebnisse der Reihen sind folgende (durchschnittliche Leseweite in Metcr):

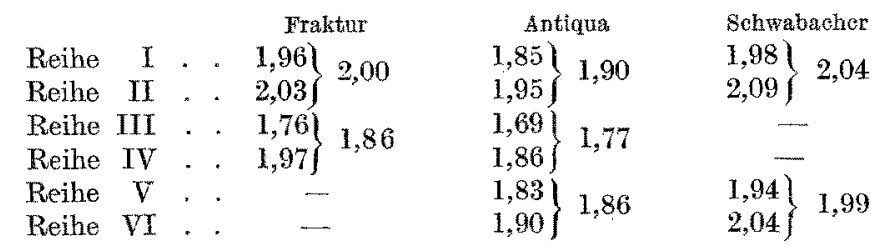


Da diese absoluten Leseweiten ohne besonderes Interesse sind, teile ich die Ergebnisse der Umrechnung nur so mit, wie sie sich an den (immor aus je 2 Reihen gemeinsam entnommenen) Verhältniszahlen darstellen. Es verhalten sich (Reihe I, II):

$$
\begin{array}{rlcl}
\text { Fraktur } & \text { Antiqua } \\
(2,00) & (1,90) & \text { Schwabacher } & =1,0: 0,95: 1,02 \\
\text { oder reduziert } & & (2,04) & =1,0: 0,92: 1,00
\end{array}
$$

Reihe III und IV gibt:

$$
\begin{aligned}
& \text { Fraktur : Antiqua } \\
& (1,86) \quad(1,77) \\
& \text { oder reduziert. } \\
& =1,0: 095 \\
& =1,0: 0,99
\end{aligned}
$$

Reihe V und VI ergibt:

$$
\begin{array}{ccc}
\begin{array}{c}
\text { Antiqua } \\
(1,86)
\end{array} & \begin{array}{c}
\text { Schwabachei } \\
(1,99)
\end{array} & =0,95: 1,02 \\
\text { oder reduziert } & & =\mathbf{0 , 9 2 : 1 , 0 0}
\end{array}
$$

Das Ergebnis ist eine völlige Ưbereinstimmung der Werte von Fraktur und Schwabacher. Dieselbe ist außerordentlich bemerkenswert angesichts der Tatsache, daß sowohl die Einzel-Kleinbuchstaben als besonders die einzelnen Großbuchstaben der Fraktur sich gegenüber der Schwabacher stark unterlegen gezeigt haben. Man sieht, daß hier ganz andere Bedingungen gelten; der etwas schmälere Schnitt erweist sich im Zusammenhange der Buchstaben augenscheinlich nicht als unvorteilhaft und die für sich allein schwer $\mathrm{zu}$ deutenden Formen der Großbuchstaben fügen sich so gut ein, daß ebenso ausgeprägte Wortbilder entstehen wie bei der Schwabacher.

Beiden Bruchschriften gegenüber ist die Unterlegenheit der Antiqua augenscheinlich, sie beträgt genau $8 \%$. Aller Wahrscheinlichkeit nach sind es die Kleinbuchstaben, welche dieses Verhältnis bedingen: Machte ihre etwas eintönige, nur aus gleich großen Rundungen und geraden Linien gebildete Form sie schon bei der Einzelablesung weniger gut kenntlich, so wirkt im Zusammenhange der Wortbilder ganz besonders die geringere Zahl von Ober- und Unterlängen ungünstig ein. Von allergrößter Bedeutung ist, um einen Buchstaben besonders hervorzuheben, sicher das lange s der Bruchschriften, welchem in den Wortbildern geradezu die Bedeutung eines Wegweisers zukommt.

Nach alledem muß ich der in der obenerwähnten Petition vertretenen Ansicht, wonach die Antiqua der Fraktur erheblich überlegen sei, entschieden widersprechen. Sind auch die Unterschiede in der Lesbarkeit nieht sehr groß, so bestehen sie doch zweifellos, und zwar zugunsten der Bruchschriften. Soviel ich übersehen kann, befinde ich mich damit in Übereinstimmung mit den Ergebnissen lesepsychologischer Untersuchungen, denen ich, wie gesagt, auch die eigentliche 
Entscheidung in der Schriftfrage überlassen möchte. Daß die vorstehenden Feststellungen aber einmal von augenärztlicher Seite gemacht worden sind, scheint mir auch deshalb wichtig, weil von altschriftfreundlichen Laien mit Vorliebe auf gelegentliche Äußerungen von Augenärzten, die der Antiqua günstig lauten sollen, hingewiesen wird.

Zum Schlusse ist es mir eine angenehme Pflicht, Herm Professor von Rohr in Jena für die Ưberlassung des Apparates und die Anregung zu den vorliegenden Untersuchungen sowie für mannigfache Unterstützung dabei meinen besten Dank auszusprechen; ebenso Herrn Professor Henker daselbst für mancherlei gewährte Hilfe und Herrn Professor Stock für die freundlich erteilte Erlaubnis, einen Teil der Untersuchungen in seiner Klinik vornehmen zu dürfen.

\section{Literatur.}

1) Triepel, Über Sehleistung bei Myopie. Arch. f. Ophthalmol, 40, 50-101. 1894. - 2) Leber, Bemerkungen über die Sehschärfe hochgradig myopischer Augen vor und nach operativer Beseitigung der Linse. Arch. f. Ophthalmol. 43, 218-251. 1897. - 3) Fuchs, Lehrbuch der Augenheilkunde, 11. Aufl., 1907. 4) Löhner, Die Sehschärfe des Menschen und ihre Prüfung. Leipzig und Wien. Franz Deuticke. 1912. - 5) Salzmann, Das Sehen in Zerstreuungsicreisen. Arch. f. Ophthalmol, 39, 83 und 40, 102. 1894; 49, 168-189. 1899. - 6) Wolffberg, Analytische Studien an Buchstaben und Zahlen zum Zweck ihrer Verwertung fiùr Sehschärfeprüfungen. Arch. f. Ophthalmol. $7 \%, 409-470$. 1910 . 7) Hess, Die Refraktion und Alkkommodation des menschlichen Auges und ihre Anomalien. Graefe-Saemischs Handbuch. IIr. Aufl. 1910. - s) Hexing, Uber die Grenzen der Sehschärfe. Ber. ü. d. Verhandl. d. Kgl, sächs. Ges. d. Wiss. zu Leipzig. Math.-Phys. Klasse. IIT. Naturw. Teil. 51, 16-24. Leipzig 1899. - $\left.{ }^{9}\right)$ Donders, Praktische Bemerkungen uber Hilfslinsen und Behschärfe. Arch. f. Ophthalmol. 18, 244. 1872. - 10) Gullstrand, Die Dioptrik des Auges (III. Aufl. des Handbuches der physiol. Optik von Helmholtz, 1. Bd., Hamburg und Leipzig 1909). - 11) v, Rohr, Die Brille als optisches Instrument. Graefe-Saemischs Handbuch. II. Aufl. 1911. - 12) Löhlein und Gebb, Zur Trage der Sehschärfebestimmung. Arch. 1 . Augenheilk. 65, 69-97 und 189--213. 1910. - 13) Landolt, Formsinn und Sehschärfe. Arch. f. Augenheilk. 55, 219-222; Die Reform der Bestimmung der Sehschärfe. Arch. f. Ophthalmol. 64, 598-611. 1906. ${ }^{14}$ ) Erdmann und Dodge, Untersuchungen über das Lesen. Halle 1898. 15) Zeitler, Tachistoskopische Untersuchungen über das Lesen. Philos. Studien 16. 1900. - 16) Schumann, Psychologie des Lesens. Psychol. Studien. 1. Abt., ㅍ. 2, 8. 30f. 1908. - ${ }^{17}$ ) MeBmer, Zur Psychologie des Lesens bei Kindern und Erwachsenen. Arch. f. d. ges. Psychol. 2, 259f. - 18) Kirsohmann, Über die Erkennbarkeit geometrischer Figuren und Schriftzeigen im indirekten Sehen. Arch. f. d. ges. Psychol. 13, 352-388. 1908. Antiqua oder Fraktur? (Monographien des Buchgewerbes Bd. I. Leipzig 1912.) - ${ }^{19}$ ) Schackwitz, Die versuchsmäßige Lösung des Sehriftstreits. 2. Aufl. K. F. Koehler. Leiprig 1912. - 20) Lobsien, Experimentelle Untersuchungen über Lesbarkeit von Fralstur und Antiqua. Beitr. zur Kinderforschung u. Heilerziehung H. 149. 1918. - ${ }^{21}$ ) Nr. 778, Reichstag, 12. Leg. Periode, II. Session 1909/11, 81. Bericht der Kommission f. d. Petitionen. 
- $\left.{ }^{22}\right)$ Guillery, Tabelle zu meinen Sehproben. Arch. f. Augenheilk. 44, 242 bis 244. 1901.

Eine sehr sorgfältige Zusammenstellung der gesamten Sehschärfeliteratur findet sich in der zitierten Arbeit von Löh ner. Sie umfaßt 632 Nummern aus den Jahren 1850-1910. Seidem sind nur noch wenige bedeutsamere Veröffentlichungen auf diesem Gebiete erschienen. Die wichtigsten dürften die folgenden sein:

Thorner, Über Sehprüfungen und Grenzen der Sehschärfe. Med. Klin. 1911, 415. - Ficker, Vergleichende Sehschärfebestimmungen. Zeitschr. f. Augenheilk. $\mathbf{2 6}$, 113. - Wolffberg, Beitrag zur Sehschärfeprüfung nach Snellen. Arch, f. Ophthalmol. 90, 249. 1915. - Gleiche n, Beitrag zur Theorie der Sehschärfe. Arch. f. Ophthalmol. 93, 303. 1917. 